\title{
Dielectric behavior of OPEFB reinforced polycaprolactone composites at X-Band frequency
}

\begin{abstract}
Short fibres are often used as reinforcing materials with thermoplastic polymers to improve the dielectric properties of the composites. In this work, the effects of oil palm empty fruit bunch (OPEFB)-fibre loading on polycaprolactone (PCL) polymer were extensively studied. The synthesis of OPEFBPCL composites were prepared via melt blend method. Theoretical and experimental analysis of electromagnetic (EMI) propagation, absorption and shielding effectiveness (SE) properties of the composite were also studied. The magnitudes of Sparameters for OPEFB fiber-reinforced PCL composites with different percentages of filler were measured by a rectangular waveguide connected with a microwave vector network analyzer (VNA) at (8-12) GHz frequency. Whilst the dielectric properties were studied using an open ended coaxial probe. The morphological characterization of the OPEFBPCL composite was carried out using X-ray diffraction and scanning electron microscopy (SEM). This work showed that XRD profile patterns slightly changed duo to the filler loading increment which result a reduction in both dielectric constant and loss factor. However, the relative permittivity of pure oil palm fibre and PCL was measured as $(1.8-\mathrm{j} * 0.08)$ and $(2.929$ $\left.\mathrm{j}^{*} 0.3242\right)$ respectively. In addition, the composition of $12.5 \mathrm{wt} \%$ filler gave the highest dielectric constant and loss factor values. However, the reflection loss increases to filler content increments at the maximum frequency of $12 \mathrm{GHz}$ - The excellent behavior of these polymer composites makes them superior nominees for microwave low absorption materials.
\end{abstract}

